\title{
Análisis comparado de la dinámica epidemiológica de la Influenza A (H1N1) en Chile
}

MAURICIO CANALS L.

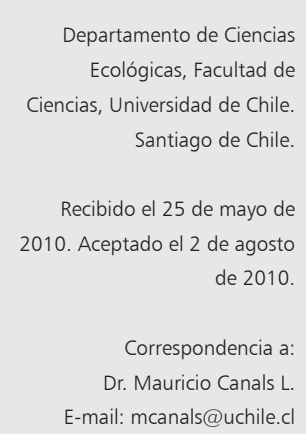

$\mathrm{E}$ n 2009 el mundo enfrentó la quinta pandemia de esta enfermedad ${ }^{1-3}$. Fue producida por el virus influenza, un virus ARN que comprende tres tipos: $\mathrm{A}, \mathrm{B}$ y $\mathrm{C}^{1}$. Todos tienen reservorio animal, en aves acuáticas, cerdos y 16 otros mamíferos ${ }^{4,5}$. Las propiedades antigénicas de Hemaglutinina $(\mathrm{H})$ y Neuraminidasa $(\mathrm{N})$ permiten la clasificación en subtipos, H1-H16 y N1-N9 ${ }^{3}$. El virus tiene gran capacidad mutagénica y de recombinación, que puede representarse por cambios menores en los antígenos $\mathrm{H}$ y $\mathrm{N}$ con el recambio generacional del virus ("drift") o cambios mayores que significan la introducción de un nuevo subtipo para el cual la población no tiene inmunidad pre-existente ("shift"). Aunque el virus influenza
$\mathrm{A}(\mathrm{H} 1 \mathrm{~N} 1)$ fue aislado en 1930 desde un cerdo ${ }^{6,7}$ su historia puede ser seguida hasta 1918 , ya que el virus de la gripe española es genéticamente similar ${ }^{8}$ y tienen un ancestro común ${ }^{9,10}$. Probablemente el $\mathrm{A}(\mathrm{H} 1 \mathrm{~N} 1)$ tiene un origen aviar y cruzó la barrera interespecífica originando la "gripe española" y sus subtipos en cerdos ${ }^{3}$. En la pandemia de 1957 el subtipo A(H2N2) se originó a partir de 5 segmentos del genoma del $\mathrm{A}(\mathrm{H} 1 \mathrm{~N} 1)$ circulante y $\mathrm{H}$, $\mathrm{N}$ y la proteína $\mathrm{PB} 1$ de origen aviar, la $\mathrm{A}(\mathrm{H} 3 \mathrm{~N} 2)$ de 1968 mantuvo 6 segmentos de la A(H2N2) y PB1 y $\mathrm{H}$ de origen aviar. En cambio la pandemia de 1977 ocurrió por un virus idéntico al de $1918^{2}$. Sin embargo, la emergencia del A(H1N1)-2009 es diferente ${ }^{7}$. En 1998 en Estados Unidos de Nortea- 
mérica se detectó en cerdos la circulación de un virus resultante del triple-reagrupamiento entre el virus de origen porcino, el $\mathrm{A}(\mathrm{H} 3 \mathrm{~N} 2)$ humano $\mathrm{y}$ un linaje norteamericano de virus aviar, dando origen al subtipo A(rH3N2). Tras la nuevas reagrupaciones con el $\mathrm{A}(\mathrm{H} 1 \mathrm{~N} 1)$ aparecieron subtipos triple-reagrupados $\mathrm{A}(\mathrm{H} 1 \mathrm{~N} 1)$ y $\mathrm{A}(\mathrm{H} 1 \mathrm{~N} 2)^{7}$. El virus actual A (H1N1)-2009 es derivado de 6 segmentos de genoma derivados del triple-reagrupado y dos segmentos del A(H1N1) de cerdo clásico ${ }^{2,3,11,12}$.

La epidemia actual se originó entre marzo y abril de 2009 en México ${ }^{13}$ identificándose el 21 de abril el nuevo virus en 2 niños del sur de California ${ }^{3}$. Con el aumento del número de casos en Norteamérica el 24 de abril de declaró la alerta de pandemia en fase 5 (Transmisión humanohumano en al menos 2 países en una región de la OMS).

La epidemia progresó rápidamente, alcanzando continuidad geográfica en Estados Unidos en 10 días $^{14}$, en el resto de América en 15 días y en el mundo en 74 días $^{14,16}$. Actualmente, se tiene el registro de 15.921 defunciones en 212 países $^{17}$. En Chile la epidemia llegó el 17 de mayo de $2009^{14,18}$. Las primeras predicciones eran preocupantes, por ser un virus nuevo, con número reproductivo y población susceptible altos ${ }^{14,19}$. Así, sólo en Santiago, el número de casos podía llegar a 2,8 millones ${ }^{14}$. Sin embargo, el comportamiento de la epidemia no fue el esperado, llamando la atención una elevada proporción de casos en jóvenes ${ }^{19,20,21}$.

En este trabajo, con el objetivo de aumentar el conocimiento de la pandemia y de racionalizar las medidas de control ante eventuales nuevos brotes (o nuevas epidemias) se analizan las características dinámicas de la pandemia de influenza $\mathrm{A}(\mathrm{H} 1 \mathrm{~N} 1)$ en Chile.

\section{Material y Métodos}

Se analizó el comportamiento de los casos confirmados diarios de Chile entre el 17 de mayo y el 9 de julio obtenidos de los reportes diarios del MINSAL $^{21}$ y los casos confirmados de los 9 países con más casos, obtenidos de las actualizaciones diarias realizadas por la $\mathrm{OMS}^{17}$ : Argentina (24/56/7), México (25/4-6/7), Estados Unidos (25/46/7), Canadá (27/4-6/7), Australia (20/5-6/7), Inglaterra (28/4-6/7), Japón (9/5-6/7), Filipinas (22/5-6/7) y Singapur (27/5-6/7).
Se estimaron los números reproductivos $\left(\mathrm{R}_{0}\right)$, las tasas de doblamiento $\left(\mathrm{T}_{2}\right)$ y las proyecciones de población susceptible e infectada al final de la epidemia $\left(\mathrm{S}^{*}\right.$ e $\left.\mathrm{I}^{*}\right)$ a partir de los datos de los 10 , de los 20 y de los 30 primeros días. El número reproductivo $\left(\mathrm{R}_{0}\right)$ se estimó a partir de la tasa de crecimiento exponencial al inicio de la epidemia $\left(\mathrm{r}_{0}\right): d y / d t=r_{0} y$, donde y es el número de casos y t el tiempo. Considerando $d t=1$ día, $d y / d t$ corresponde al número de casos nuevos diarios (incidencia diaria). Entonces $r_{0}$ se obtuvo del ajuste de una recta de regresión entre los casos totales y la incidencia diaria $^{22}$. A partir de $r_{0}$ se obtuvo $R_{0}$ con: $R_{0}=1+$ $\tau \cdot \mathrm{r}_{0}$, donde es el tiempo promedio entre la adquisición de la enfermedad y el contagio (período serial). También se calculó el valor de $R_{0}$ bajo el supuesto de la existencia de supercontagiantes $\left(R_{0 \mathrm{~s}}\right): R_{0 \mathrm{~s}}=1+\tau \cdot \mathrm{r}_{0}+f(1-f)\left(\tau \cdot \mathrm{r}_{0}\right)^{2}$ donde $f$ es la razón entre el período infeccioso y el período serial. Estimaciones previas para otras epidemias del subtipo A ( $\mathrm{H} 1 \mathrm{~N} 1)$ sugieren un período latente de 1,9 días, un período infeccioso de 4,1 días y un período serial $\tau=6$ días ${ }^{14,18,23}$. Se estimó la fracción de infectados $\left(I^{*}\right)$ y la fracción final de susceptibles libres de enfermedad al final de la epidemia $\left(S^{*}=1-I^{*}\right)$ con: $R_{0}=\frac{\ln S^{*}}{S^{*}-1} \quad{ }^{14,24,25}$. El tiempo de doblamiento se obtuvo a partir de $T_{2}=\frac{\ln (2)}{r_{0}}{ }^{14}$.

La dinámica poblacional básica al inicio de una epidemia en una población de tamaño $\mathrm{N}$ se puede expresar mediante $d y / d t=\beta(N-y) y-(\gamma+\psi) y=$ $\beta(N-y) y-\phi y=(\beta N-\phi) y-\beta y^{2}[1]^{18}$, donde $\beta$ es el coeficiente de transmisión (o transmisibilidad) $\gamma$ la tasa de recuperación, $\psi$ la tasa de mortalidad y $\Phi=$ $\gamma+\psi$. La relación entre la incidencia diaria $(d y / d t)$ y el número total de casos acumulados $(y)$ es una parábola, lo que permitió mediante regresión cuadrática estimar el valor de la transmisibilidad $(\beta)$.

La solución de la ecuación [1] conduce a la curva de aumento de casos totales en una epidemia, y su derivada a la curva epidémica en una población cerrada:

$y^{\prime}(t)=\frac{a^{2} C \cdot e^{a t}}{\left[1+\beta C e^{a t}\right]^{2}} \quad$ [2] donde $a=\beta N-\phi y C=$ $y_{0} /\left(a-\beta y_{0}\right)$, con $y_{0}$ el número inicial de casos ${ }^{24-26}$. Se estudió el ajuste de esta curva para Chile con diferentes condiciones iniciales y se compararon los valores con la incidencia observada con análisis 
de correlación. Se realizaron simulaciones utilizando la versión discreta de la ecuación [1]: $y_{t+1}=y_{t}+$ $[a-\beta y] \cdot y=y_{t}+(a / K)\left[K-y_{t}\right] \cdot y^{18}$, donde $K=$ $a / \beta$ representa la capacidad de carga del sistema. Se usaron los supuestos $R_{0}=1,5$ y $R_{0}=2$ y se estimó la población susceptible. Esta en un estrato etario " $x$ " $\left(S_{x}\right)$ se estimó a partir de:

$S_{x}=\frac{\left[\frac{P E_{x}}{P P_{x}}\right]}{\left[\frac{P E_{x}}{P P_{x}}\right]_{\max }} \cdot P_{x}$, donde $\left[\frac{P E_{x}}{P P_{x}}\right]$ representa el cociente entre la proporción de enfermos a la edad $\mathrm{x}\left(P E_{x}\right)$ y la proporción de individuos de edad $\mathrm{x}$ en la población $\left(\mathrm{PP}_{x}\right),\left[\begin{array}{c}P E_{x} \\ P P_{x}\end{array}\right]_{\max }$ es el máximo de estos cocientes y $P_{x}$ representa el número de individuos de edad x en la población. El número total de susceptibles corresponde a la suma de los $S_{x}$. Las poblaciones fueron obtenidas del MINSAL ${ }^{21}$. Se consideraron diferentes razones de notificación de casos ( $\mathrm{n}=$ casos notificados/casos reales): $1 / 10$; $1 / 3 ; 1 / 2$ y $8 / 10$.

\section{Resultados}

Los números reproductivos fueron disminuyendo desde $R_{0} \approx 2$, hasta entre 1,37 y 1,82 a los 30 días, en todos los países. Los valores más bajos se encontraron en Inglaterra, Japón, Filipinas y Singapur (Figura 1). Los períodos de doblamiento aumentaron desde aproximadamente 3 días hasta entre 5 y 8 días (Tabla 1). De acuerdo a esto, las proporciones de infectados al final de la epidemia variarían entre $58 \%$ y $78,5 \%$.

El ajuste de las curvas de incidencia vs casos fue buena para todos los países, $\mathrm{R}^{2}$ entre 0,48 y 0,99 (Tabla 2, Figura 2). Los coeficientes de transmisión variaron desde 2 hasta 132 casos nuevos/día x $10^{6}$ susceptibles (Figura 3). El ajuste de la curva de casos diarios vs casos totales en Chile fue adecuado $\left(\mathrm{dy} / \mathrm{dt}=0,11887 \mathrm{y}-0,000012 \mathrm{y}^{2} ; \mathrm{F}_{2,52}=145,72, \mathrm{R}^{2}=\right.$ $0,84, \mathrm{p}<<0,001)$, sin embargo, la curva epidémica para la condición inicial de 2 casos presentó un mal ajuste $\left(\mathrm{r}=0,14, \mathrm{~F}_{1,52}=1,1, \mathrm{p}=0,29\right)$, pero los ajustes fueron buenos para 200 a 250 casos iniciales $(r=0,667$ y $r=0,671$; Figura 4). La población total de susceptibles en todo Chile se estimó en 6.717.807 individuos (Tabla 3). Las simulaciones describieron una curva epidémica más precoz y

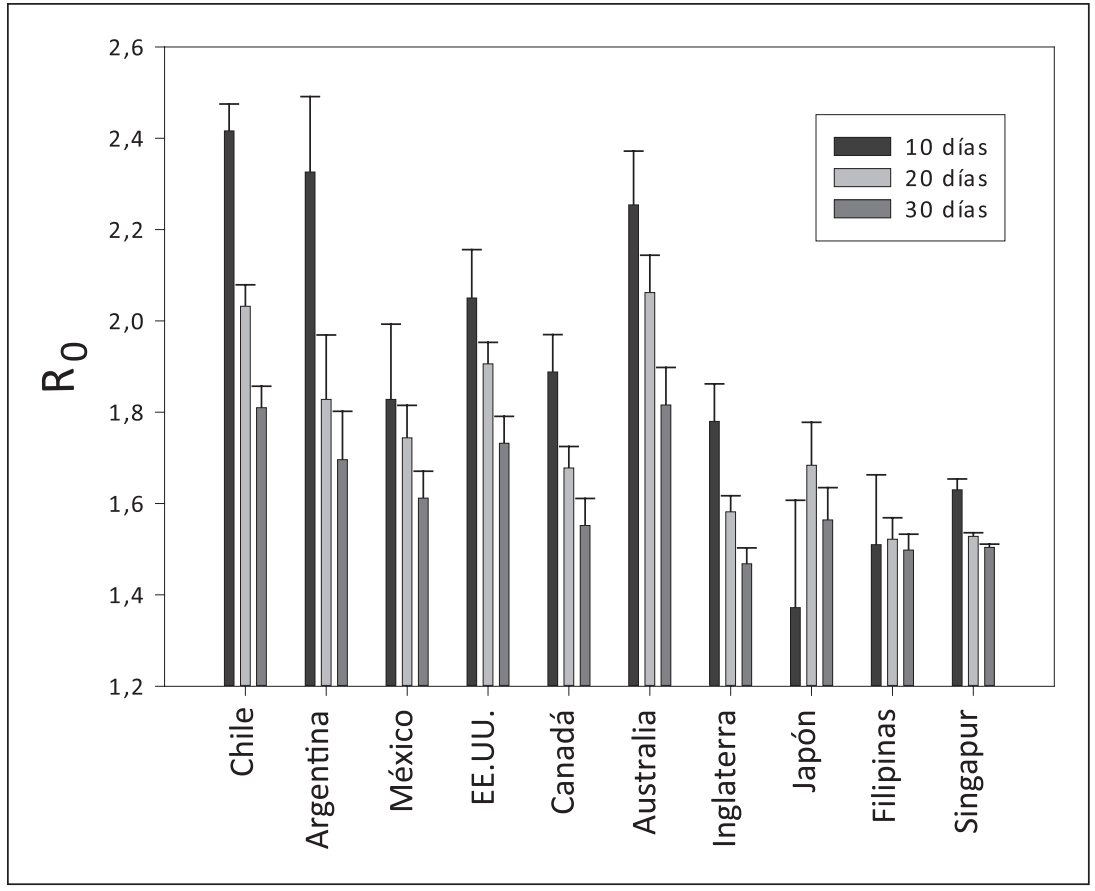

Figura 1. Números reproductivos estimados a los 10, 20 y 30 primeros días de la epidemia A(H1N1) 2009 en los 10 países con más casos confirmados. 
ARTÍCULO ESPECIAL

Dinámica epidemiológica de la Influenza A (H1N1) en Chile - M. Canals L.

Tabla 1. Tasas instantáneas de crecimiento $\left(r_{0}\right)$, números reproductivos $\left(R_{0}\right)$ sin y con supuesto de existencia de supercontagiantes $\left(R_{0 s}\right)$, proporción estimada de susceptibles $\left(S^{*}\right)$ e infectados $\left(I^{*}\right)$ al final de la epidemia y tiempos de doblamiento $\left(T_{2}\right)$ para los 10 países con más casos de influenza A (H1N1) durante 2009, estimados a partir de los 10, 20 y 30 primeros días de epidemia

\begin{tabular}{|c|c|c|c|c|c|c|}
\hline \multicolumn{7}{|c|}{10 días } \\
\hline & $r_{0}$ & $\mathbf{R}_{0}$ & $\mathbf{R}_{\text {os }}$ & $\mathbf{S}^{*}$ & $\mathbf{I}^{*}$ & $\mathbf{T}_{2}$ \\
\hline Chile & $\begin{array}{c}0,236 \\
(0,226-0,246)\end{array}$ & $\begin{array}{c}2,418 \\
(2,357-2,475)\end{array}$ & 2,849 & 0,070 & 0,930 & 2,937 \\
\hline Argentina & $\begin{array}{c}0,221 \\
(0,194-0,248)\end{array}$ & $\begin{array}{c}2,326 \\
(2,161-2,491)\end{array}$ & 2,706 & 0,085 & 0,915 & 3,136 \\
\hline México & $\begin{array}{c}0,138 \\
(0,111-0,165)\end{array}$ & $\begin{array}{c}1,828 \\
(1,663-1,993)\end{array}$ & 1,976 & 0,210 & 0,790 & 5,023 \\
\hline Estados Unidos & $\begin{array}{c}0,175 \\
(0,157-0,193)\end{array}$ & $\begin{array}{c}2,050 \\
(1,944-2,156)\end{array}$ & 2,288 & 0,140 & 0,860 & 3,961 \\
\hline Canadá & $\begin{array}{c}0,148 \\
(0,134-0,162)\end{array}$ & $\begin{array}{c}1,888 \\
(1,806-1,970)\end{array}$ & 2,058 & 0,190 & 0,810 & 4,683 \\
\hline Australia & $\begin{array}{c}0,209 \\
(0,189-0,229)\end{array}$ & $\begin{array}{c}2,254 \\
(2,136-2,372)\end{array}$ & 2,594 & 0,095 & 0,905 & 3,316 \\
\hline Inglaterra & $\begin{array}{c}0,130 \\
(0,116-0,144)\end{array}$ & $\begin{array}{c}1,780 \\
(1,698-1,862)\end{array}$ & 1,911 & 0,225 & 0,775 & 5,332 \\
\hline Japón & $\begin{array}{c}0,062 \\
(0,023-0,101)\end{array}$ & $\begin{array}{c}1,372 \\
(1,137-1,607)\end{array}$ & 1,402 & 0,485 & 0,515 & 11,180 \\
\hline Filipinas & $\begin{array}{c}0,085 \\
(0,060-0,110)\end{array}$ & $\begin{array}{c}1,510 \\
(1,357-1,663)\end{array}$ & 1,566 & 0,375 & 0,625 & 8,155 \\
\hline Singapur & $\begin{array}{c}0,105 \\
(0,101-0,109)\end{array}$ & $\begin{array}{c}1,630 \\
(1,606-1,654)\end{array}$ & 1,716 & 0,300 & 0,700 & 6,601 \\
\hline \multicolumn{7}{|c|}{20 días } \\
\hline Chile & $\begin{array}{c}0,172 \\
(0,164-0,180)\end{array}$ & $\begin{array}{c}2,032 \\
(1,985-2,079)\end{array}$ & 2,262 & 0,195 & 0,805 & 4,030 \\
\hline Argentina & $\begin{array}{c}0,138 \\
(0,114-0,162)\end{array}$ & $\begin{array}{c}1,828 \\
(1,663-1,993)\end{array}$ & 1.976 & 0,210 & 0,790 & 5,023 \\
\hline México & $\begin{array}{c}0,124 \\
(0,112-0,136)\end{array}$ & $\begin{array}{c}1,744 \\
(1,673-1,815)\end{array}$ & 1,864 & 0,245 & 0,755 & 5,590 \\
\hline Estados Unidos & $\begin{array}{c}0,151 \\
(0,143-0,159)\end{array}$ & $\begin{array}{c}1,906 \\
(1,859-1,953)\end{array}$ & 2,083 & 0,180 & 0,820 & 4,590 \\
\hline Canadá & $\begin{array}{c}0,113 \\
(0,105-0,121)\end{array}$ & $\begin{array}{c}1,678 \\
(1,631-1,725)\end{array}$ & 1,777 & 0,275 & 0,725 & 6,134 \\
\hline Australia & $\begin{array}{c}0,177 \\
(0,163-0,191)\end{array}$ & $\begin{array}{c}2,062 \\
(1,980-2,144)\end{array}$ & 2,306 & 0,190 & 0,810 & 3,916 \\
\hline Inglaterra & $\begin{array}{c}0,097 \\
(0,091-0,103)\end{array}$ & $\begin{array}{c}1,582 \\
(1,547-1,617)\end{array}$ & 1,655 & 0,300 & 0,700 & 7,146 \\
\hline Japón & $\begin{array}{c}0,114 \\
(0,098-0,130)\end{array}$ & $\begin{array}{c}1,684 \\
(1,590-1,778)\end{array}$ & 1,785 & 0,325 & 0,675 & 6,080 \\
\hline Filipinas & $\begin{array}{c}0,087 \\
(0,079-0,095)\end{array}$ & $\begin{array}{c}1,522 \\
(1,475-1,569)\end{array}$ & 1,581 & 0,370 & 0,630 & 7,967 \\
\hline Singapur & $\begin{array}{c}0,088 \\
(0,087-0,089)\end{array}$ & $\begin{array}{c}1,528 \\
(1,520-1,536)\end{array}$ & 1,588 & 0,365 & 0,635 & 7,877 \\
\hline
\end{tabular}


Tabla 1. Tasas instantáneas de crecimiento $\left(r_{0}\right)$, números reproductivos $\left(R_{0}\right)$ sin y con supuesto de existencia de supercontagiantes $\left(R_{0 s}\right)$, proporción estimada de susceptibles $\left(S^{*}\right)$ e infectados $\left(I^{*}\right)$ al final de la epidemia y tiempos de doblamiento $\left(T_{2}\right)$ para los 10 países con más casos de influenza $A$ (H1N1) durante 2009, estimados a partir de los 10, 20 y 30 primeros días de epidemia (continuación)

\begin{tabular}{|c|c|c|c|c|c|c|}
\hline & & & 30 días & & & \\
\hline & $r_{0}$ & $\mathbf{R}_{0}$ & $\mathbf{R}_{\text {os }}$ & $\mathbf{S}^{*}$ & $\mathbf{I}^{*}$ & $\mathbf{T}_{2}$ \\
\hline Chile & $\begin{array}{c}0,135 \\
(0,127-0,143)\end{array}$ & $\begin{array}{c}1,810 \\
(1,763-1,857)\end{array}$ & 1,952 & 0,215 & 0,785 & 5,134 \\
\hline Argentina & $\begin{array}{c}0,116 \\
(0,098-0,134)\end{array}$ & $\begin{array}{c}1,696 \\
(1,590-1,802)\end{array}$ & 1,801 & 0,265 & 0,735 & 5,975 \\
\hline México & $\begin{array}{c}0,102 \\
(0,092-0,112)\end{array}$ & $\begin{array}{c}1,612 \\
(1,553-1,671)\end{array}$ & 1,693 & 0,310 & 0,690 & 6,796 \\
\hline Estados Unidos & $\begin{array}{c}0,122 \\
(0,112-0,132)\end{array}$ & $\begin{array}{c}1,732 \\
(1,673-1,791)\end{array}$ & 1,848 & 0,250 & 0,750 & 5,682 \\
\hline Canadá & $\begin{array}{c}0,092 \\
(0,082-0,102)\end{array}$ & $\begin{array}{c}1,552 \\
(1,493-1,611)\end{array}$ & 1,618 & 0,350 & 0,650 & 7,534 \\
\hline Australia & $\begin{array}{c}0,136 \\
(0,122-0,150)\end{array}$ & $\begin{array}{c}1,816 \\
(1,734-1,898)\end{array}$ & 1,960 & 0,215 & 0,785 & 5,097 \\
\hline Inglaterra & $\begin{array}{c}0,078 \\
(0,072-0,084)\end{array}$ & $\begin{array}{c}1,468 \\
(1,433-1,503)\end{array}$ & 1,515 & 0,440 & 0,560 & 8,887 \\
\hline Japón & $\begin{array}{c}0,094 \\
(0,082-0,106)\end{array}$ & $\begin{array}{c}1,564 \\
(1,493-1,635)\end{array}$ & 1,633 & 0,340 & 0,660 & 7,374 \\
\hline Filipinas & $\begin{array}{c}0,083 \\
(0,077-0,089)\end{array}$ & $\begin{array}{c}1,498 \\
(1,463-1,533)\end{array}$ & 1,552 & 0,420 & 0,580 & 8,351 \\
\hline Singapur & $\begin{array}{c}0,084 \\
(0,083-0,085)\end{array}$ & $\begin{array}{c}1,504 \\
(1,497-1,511)\end{array}$ & 1,559 & 0,380 & 0,620 & 8,252 \\
\hline
\end{tabular}

Tabla 2. Estimaciones de los coeficientes de transmisión ( $\beta$ ) de la influenza A (H1N1) de los 10 países con más casos en 2009 . $F$ es el valor del estadígrafo, $R^{2}$ es el coeficiente de determinación y p es la probabilidad de Error de tipo I (p-value)

\begin{tabular}{|lcccc|}
\hline & \multicolumn{1}{c}{$\boldsymbol{\beta}$} & $\mathbf{F}$ & $\mathbf{R}^{\mathbf{2}}$ & $\mathbf{p}$ \\
Chile & 0,000012 & 145,7 & 0,84 & $<<0,001$ \\
\hline Argentina & 0,000030 & 32,5 & 0,66 & $<<0,001$ \\
\hline México & 0,000006 & 20,1 & 0,54 & $<<0,001$ \\
Estados Unidos & 0,000002 & 49,8 & 0,74 & $<<0,001$ \\
Canadá & 0,000012 & 26,5 & 0,59 & $<<0,001$ \\
Australia & 0,000011 & 51,5 & 0,83 & $<<0,001$ \\
Inglaterra & 0,000007 & 77,1 & 0,78 & $<<0,001$ \\
Japón & 0,000010 & 15,1 & 0,48 & $<0,001$ \\
Filipinas & 0,000026 & 194,8 & 0,99 & $<<0,001$ \\
Singapur & 0,000132 & 63,4 & 0,90 & $<<0,001$ \\
\hline
\end{tabular}




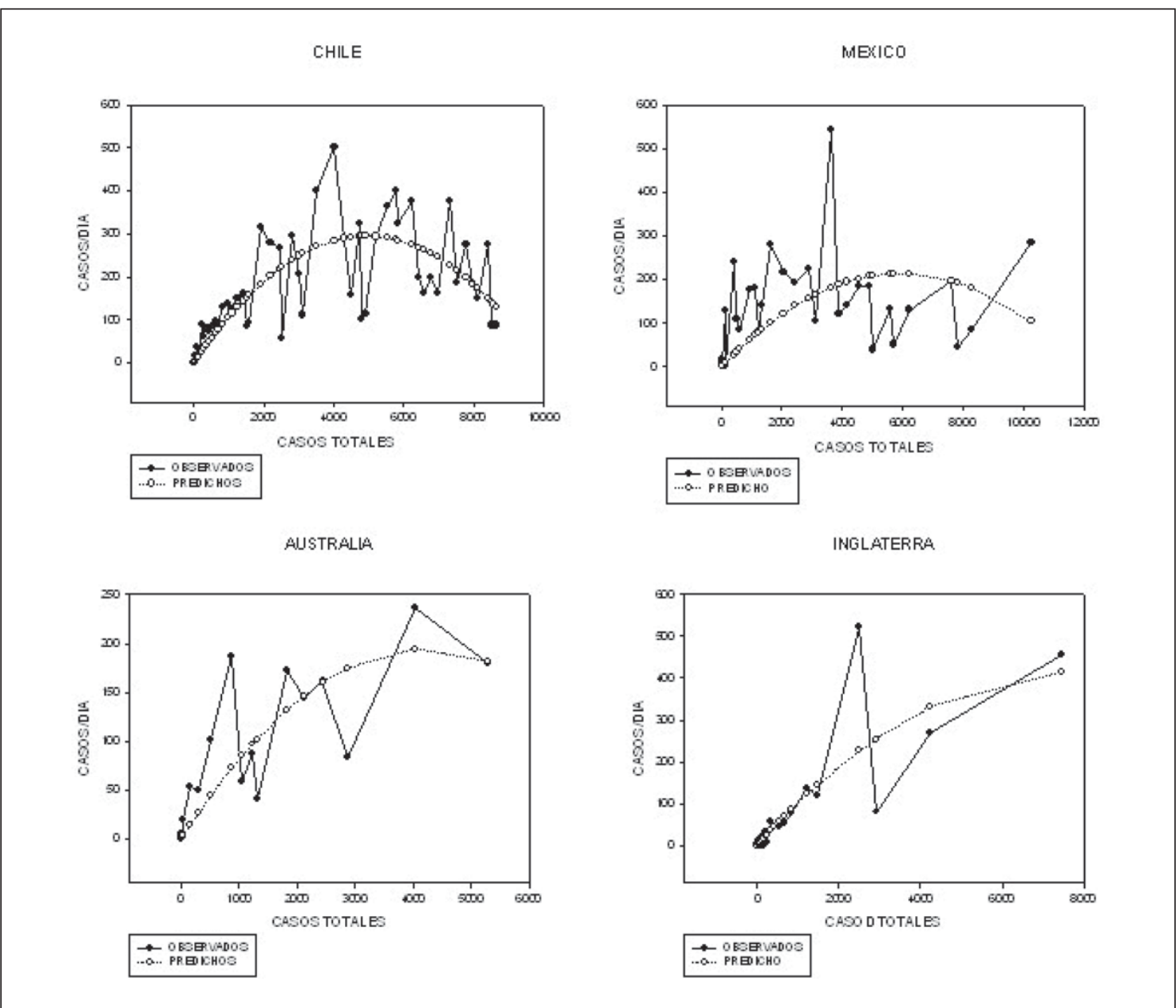

Figura 2. Ajustes de las curvas incidencia vs casos totales en cuatro países representativos con alto número de casos.

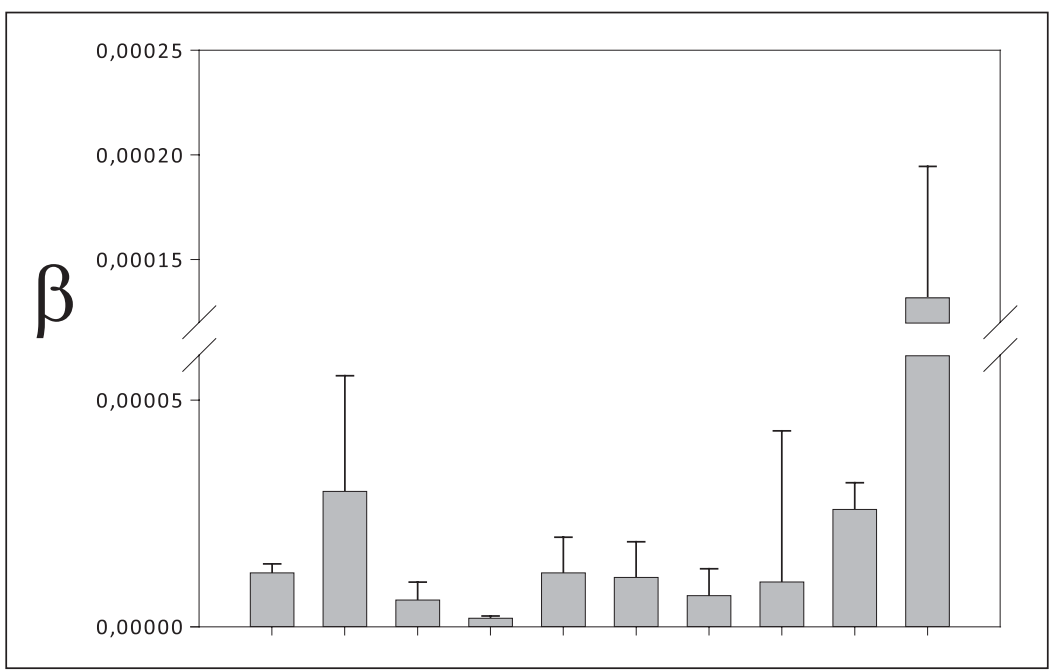

Rev Med Chile 2010; 138: 1186-1196
Figura 3. Coeficiente de transmisión ( $\beta$ ) estimados a los 30 primeros días de la epidemia A(H1N1) 2009 en los 10 países con más casos confirmados. 


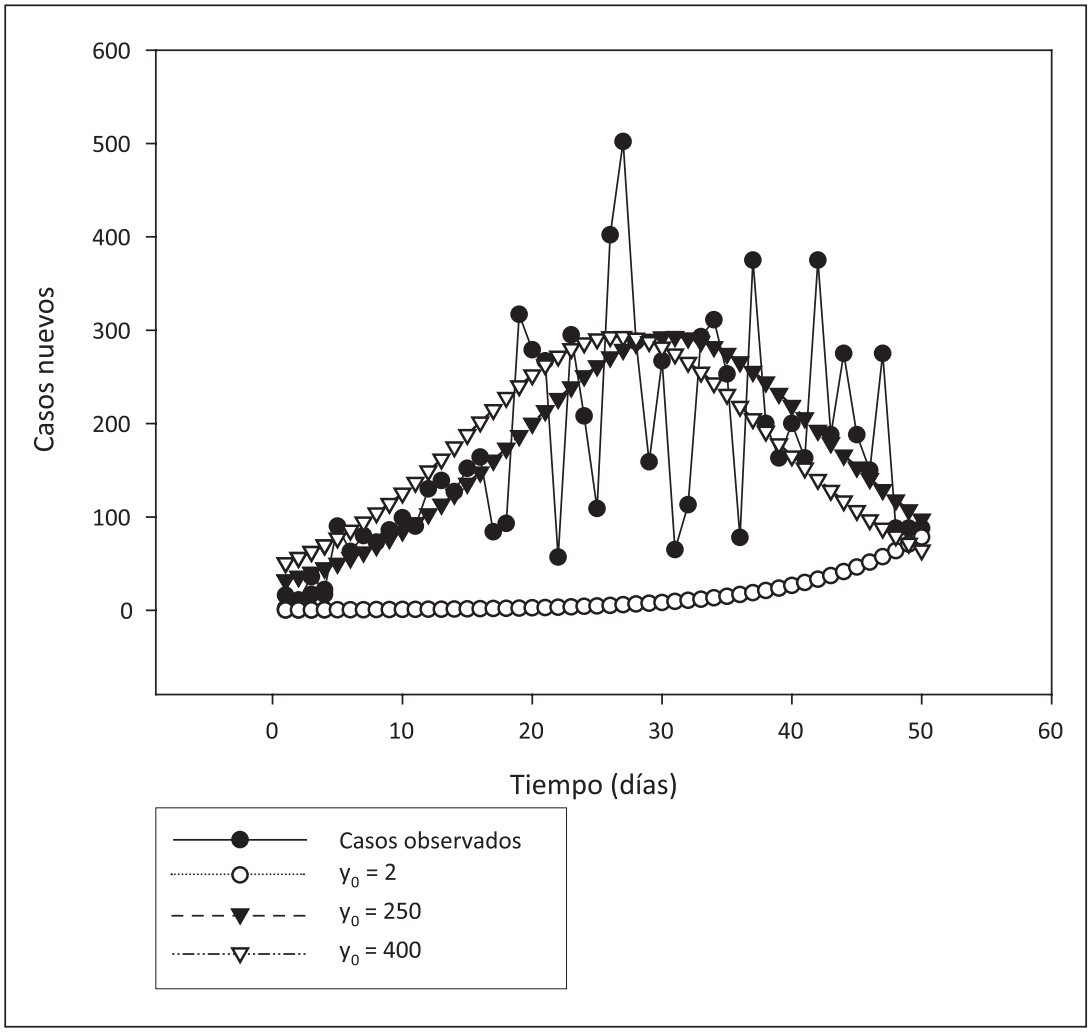

Figura 4. Ajustes de la curvas epidémicas a los casos reportados y confirmados de influenza $\mathrm{A}(\mathrm{H} 1 \mathrm{~N} 1)$ en Chile 2009 para diferentes condiciones iniciales $\left(y_{0}\right)$.

Tabla 3. Estimación de la población susceptible en cada estrato etario $\left(\mathrm{S}_{\mathrm{x}}\right)$, basada en la distribución de casos de influenza, la población en cada estrato $\left(P_{x}\right)$ y proporciones de enfermos $\left(\mathrm{PE}_{\mathrm{x}}\right)$ y población $\left(\mathrm{PP}_{\mathrm{x}}\right)$ en cada estrato (ver texto)

\begin{tabular}{|lcccccc|}
\hline $\begin{array}{l}\text { Intervalo } \\
\text { etario } \\
\text { (años) }\end{array}$ & Casos & Px & PEx & PPx & {$\left[\frac{P E_{x}}{P P_{x}}\right]$} & Sx \\
\hline $0-4$ & 1.365 & 1246153 & 0,145 & 0,074 & 1,967 & 972820,5 \\
\hline $5-9$ & 1.764 & 1255622 & 0,187 & 0,074 & 2,523 & 1257183,4 \\
\hline $10-14$ & 1.879 & 1360847 & 0,199 & 0,080 & 2,480 & 1339142,7 \\
\hline $15-19$ & 944 & 1483285 & 0,100 & 0,088 & 1,143 & 672778,4 \\
\hline $20-24$ & 578 & 1434300 & 0,061 & 0,085 & 0,724 & 411934,3 \\
\hline $25-29$ & 520 & 1290814 & 0,055 & 0,076 & 0,723 & 370598,3 \\
\hline $30-34$ & 389 & 1183619 & 0,041 & 0,070 & 0,590 & 277236,0 \\
\hline $35-39$ & 448 & 1236753 & 0,048 & 0,073 & 0,651 & 319284,7 \\
\hline $40-44$ & 334 & 1237907 & 0,035 & 0,073 & 0,485 & 238038,1 \\
\hline $45-49$ & 310 & 1217407 & 0,033 & 0,072 & 0,457 & 220933,6 \\
\hline 50 y más & 895 & 3982166 & 0,094 & 0,235 & 0,403 & 637856,7 \\
\hline Total & 9.426 & 16928873 & 1,000 & 1,00 & & 6717806,8 \\
\hline
\end{tabular}




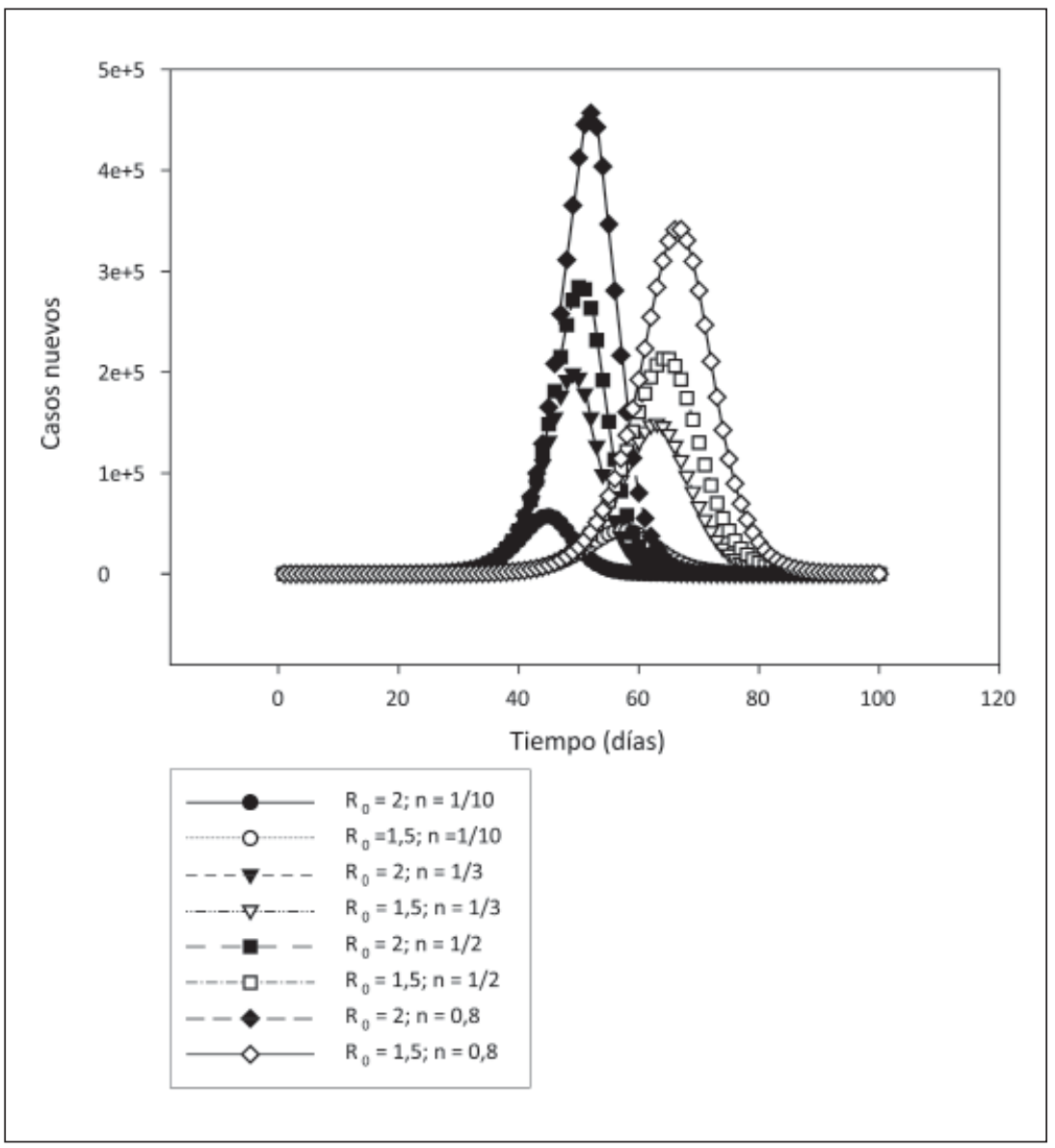

Figura 5. Simulaciones de la epidemia de influenza en Chile 2009, bajo supuestos de diferentes números reproductivos $\left(R_{0}\right)$ y diferentes proporciones de notificación ( $\mathrm{n}$ ). con mayor número de casos nuevos por día para $R_{0}=2$. Al comparar con la situación real en Chile (Figura 5) la curva es muy semejante en su forma pero muy inferior en el número de casos, sólo asemejándose a la curva con $n=1 / 10$.

\section{Discusión}

Este estudio se basó en los casos informados por la OMS y MINSAL y tiene los sesgos propios de la confirmación, notificación e información de casos. No todos los casos se confirman y notifican y la información puede ir retrasada respecto a la ocurrencia de los casos. Pueden ocurrir acumulaciones de casos que son notificados todos de una vez y que producen irregularidades artificiales en las curvas de casos, apreciables en los gráficos de casos nuevos vs casos totales (Figura 2). A pesar de esto, las estimaciones de los números reproductivos y los tiempos de doblamiento fueron consistentes y coinciden con estimaciones previas en Chile ${ }^{14} \mathrm{y}$ en Estados Unidos ${ }^{19}$. La evolución del número reproductivo es hacia la reducción y lo contrario ocurre con el tiempo de doblamiento. Los valores entre 1,4 y 1,8 para el número reproductivo y entre 5 y 11 días para el tiempo de doblamiento a los treinta días de epidemia son consistentes con estimaciones previas para $\mathrm{A}(\mathrm{H} 1 \mathrm{~N} 1)^{23}$. La caída del número reproductivo se explica por la siguiente relación: $R_{0}=\frac{\beta N}{(\gamma+\psi)}$, donde $\beta N$ representa la "fuerza de infección" y $1 /(\gamma+\psi)$ el tiempo medio de infección ${ }^{15,24-26}$. Mientras el último factor es prácticamente constante, la fuerza de infección 
va decreciendo ya que los susceptibles van disminuyendo ${ }^{26-28}$. El coeficiente de transmisión se puede expresar como el producto entre la tasa de contacto agente-hospedero $(b)$ y la probabilidad que dicho contacto resulte en infección $P(i / c)$ : $\beta=b \cdot P(i / c)^{24-29}$. La tasa de contacto corresponde a la tasa de encuentros entre casos y susceptibles, y depende de factores sociales como el hacinamiento en espacios públicos y la probabilidad de que el contacto resulte en infección depende de la temperatura, dosis infectante e inmunidad del hospedero. El coeficiente de transmisión disminuye por la caída de las tasas de contacto casos-susceptibles al enfermarse e inmunizarse gran cantidad de la población $^{30}$. La variabilidad de $\beta$ se encuentra de acuerdo a lo esperable ya que se trata de muy diferentes países, en diferentes estaciones del año $y$ en diferentes densidades de población.

En Chile el ajuste de la curva epidémica fue bueno sólo cuando se estableció una condición inicial de más de 200 casos. Esto significa que el ascenso veloz de casos no es explicado por los parámetros derivados de la misma curva. Esto tiene dos posibles explicaciones: i) que al inicio de la epidemia $R_{0}$ y $\beta$ son mucho mayores que lo estimado para 10, 20 y 30 días, y o ii) que cuando se reportó el primer caso, en realidad ya había muchos más. Aunque ambas explicaciones son posibles, el aumento de casos nuevos a partir de dos casos habría sido mucho más lento.

Frente a un nuevo subtipo de influenza, la población de susceptibles debería ser toda la población. Sin embargo, esto no parece ser así. En ensayos de microneutralización en sueros almacenados de individuos pre y post-vacuna para la influenza estacional ${ }^{31}$ se encontró que mientras los niños no presentaban reacción cruzada pre- o post-vacuna con el subtipo A(H1N1), los jóvenes entre 18 y 40 años presentaban reactividad cruzada en $6 \%$ pre- y $7 \%$ post-vacuna, entre 40 y 64 años presentaban $9 \%$ pre- y $25 \%$ post-vacuna y sobre 60 años presentaban los elevados porcentajes 33\% pre- y $43 \%$ post-vacuna. Por otra parte, la epidemia ha mostrado una desproporción de casos en jóvenes ${ }^{19,21}$. Nuestra estimación de la población susceptible se basó en la suposición que en el estrato etario que tuvo una mayor tasa de ataque, el $100 \%$ son susceptibles y que en el resto de los estratos etarios la proporción de susceptibles desciende con la tasa de ataque. Sin embargo, podría ocurrir que el estrato etario con mayor proporción de casos tenga más susceptibles pero no necesariamente todos y nuestro valor podría ser una sobre estimación. Sin embargo, si en nuestra población aplicamos los porcentajes de reacción cruzada con el virus, el número de susceptibles obtenido es aún mayor. Así, ¿por qué no se enfermaron todos?. En una epidemia nunca se enferman todos los susceptibles, porque a medida que aumenta el número de casos va disminuyendo el número de susceptibles y aumentando el número de recuperados (inmunizados) produciendo una "dilución de los susceptibles en los casos"26 que reduce la tasa de contacto casos-susceptibles. Los susceptibles al final de una epidemia dependen del número reproductivo, y en esta epidemia debieron ser cercanos al $20 \%$, lo que implica que $80 \%$ de la población susceptible debió adquirir la enfermedad, lo que es muy elevado. Aun utilizando cálculos que proponen entre 12 y $30 \%$ de la población haría influenza clínica durante una epidemia ${ }^{20}$, el número de casos habría sido muy alto.

En nuestras simulaciones, la situación más parecida a lo que ocurrió en Chile fue la epidemia más pequeña, que tenía el supuesto que 1 de cada 10 casos reales se llegó a confirmar. En esta situación se habrían confirmado más de 600.000 casos, lo que concuerda con las dosis de antiviral entregadas por el MINSAL pero excede a los casos confirmados (375.000). Aunque no se tienen estimaciones de la proporción de casos notificados en una epidemia de influenza, el supuesto que sólo uno de cada 10 casos llega a notificación no parece ser adecuado, por lo que es válido preguntar ¿por qué hubo tan pocos casos?. Hay algunas respuestas posibles: i) que la población susceptible en realidad haya sido mucho menor; ii) que la notificación y confirmación de casos sea efectivamente baja e iii) que no se hayan agotado todos los susceptibles en este primer brote. Una baja población susceptible implicaría que la experiencia antigénica previa ante vacunas y otras epidemias $\mathrm{A}(\mathrm{H} 1 \mathrm{~N} 1)$ habrían conferido inmunidad a gran parte de la población, lo que no ha sido demostrado y sólo podría ser parte de la explicación a la luz de las cifras de reacciones cruzadas ${ }^{31}$. Una baja notificación podría producirse ante gran cantidad de casos subclínicos o banales que hicieran innecesaria la confirmación y notificación. Esto podría ser consecuencia o estar asociado al punto anterior. Que no se hayan agotado los susceptibles en este primer brote es altamente probable ya que, aunque si bien en una 
epidemia es posible predecir la proporción de población que enfermará, no es posible decidir cuándo lo hará. Así las pandemias previas han ocurrido en dos o más brotes y sin clara estacionalidad $^{32}$. Por ejemplo, en la pandemia española (1918) alrededor de 65\% de la mortalidad ocurrió fuera de estación en Copenhague.

El inicio de un brote tiene relación con el umbral necesario de susceptibles que rodea un grupo inicial de $\operatorname{casos}^{24-29}$. En el caso de enfermedades de transmisión respiratoria, como la influenza, son relevantes el agrupamiento de personas en colegios, consultorios y medios de transporte y las temperaturas bajas de invierno ${ }^{29-30}$.

Es posible esperar nuevos brotes de influenza A(H1N1) mientras hayan susceptibles en número suficiente y un grupo de casos iniciales. El umbral para una población $\left(\mathrm{N}_{\mathrm{T}}\right)$ se puede estimar con $N_{T}=\frac{\gamma+\psi}{\beta}{ }^{24-29}$, lo que para Chile, considerando $\gamma \approx 0,17, \psi \approx 0$ y $\beta \approx 0,000012$ es $N_{T} \approx 14.000$ susceptibles. Así podría desencadenarse un brote en localidades pequeñas e incluso en espacios públicos.

Se desprende de este análisis que aún debería hacerse un esfuerzo en prevención, inmunizando a la población. La proporción de población a inmunizar (p) para erradicar la enfermedad está dada por: $p=1-1 / R_{0}^{24,25}$ que para el caso de Chile sería el $44,75 \%$ de los susceptibles.

\section{Referencias}

1. Acha PN, Cifres B. Zoonosis y enfermedades transmisibles comunes al hombre y los animales. II Clamidiosis, rickettsiosis y virosis. OPS publicación científica y técnica 2003; 580: 329-46.

2. Ebrahim GJ. Swine Flu (S-OIV) Pandemic. Journal of Tropical Pediatrics 2010; 56: 1-3.

3. Sullivan SJ, Jacobson RM, Dowdle WR, Poland GA. 2009 H1N1 Influenza. Mayo Clin Proc 2010; 85: 64-76.

4. Neumann G, Noda T, Kawaoka Y. Emergence and pandemic potential of swine-origin H1N1 influenza virus. Nature 2009; 459 (7249): 931-9.

5.- Gathered D. The $2009 \mathrm{H} 1 \mathrm{N1}$ influenza outbreak in its historical context. J Clin Virol 2009; 45: 174-8.

6. Shope RE. The incidence of neutralizing antibodies for swine influenza virus in the sera of human beings of different ages. J Exp Med 1936; 63: 669-84.
7. Garten RJ, Davis CT, Russell CA, et al. Antigenic and genetic characteristics of swine-origin $2009 \mathrm{~A}(\mathrm{H} 1 \mathrm{~N} 1)$ influenza viruses circulating in humans. Science 2009; 325 (5937): 197-201.

8. Tumpey TM, Basler CF, Aguilar PV, Zeng H, Solorzano A, Swayne DE, et al. Characterization of the reconstructed 1918 spanich influenza pandemic virus Science 2005; 310: 77-80.

9. Gorman OT, Bean WJ, Kawaoka Y, Donatelli I, Guo YJ, Webster RG. Evolution of influenza A virus nucleoprotein genes: implications for the origins of H1N1 human and classical swine viruses J Virol 1991; 65: 3704-14.

10. Reid AH, Taubenberger JK. J Genet Virol 2003; 84: 2285.

11. Zimmer SM, Burke DS. Historical perspective - Emergence of influenza $\mathrm{A}(\mathrm{H} 1 \mathrm{~N} 1)$ viruses. $\mathrm{N}$ Engl J Med 2009; 361: 279-85.

12. Novel Swine-Origin Influenza A(H1N1) Virus Investigation Team. Emergence of a novel swine-origin influenza A(H1N1) virus in humans. N Engl J Med 2009; 360: 2605-15.

13. Avila-Agüero ML. Influenza A(H1N1): un riesgo global. Acta Med Costarricense 2009; 51: 132-4.

14. Canals M. Inicio de la pandemia AH1N1: Algebra, cálculo y geometría del contagio. Rev Med Chile 2009; 137: 852-856.

15. Canals M, Labra F. Análisis no lineal de la dinámica de enfermedades infecciosas en Chile. Rev Med Chile 1999; 127: 1086-92.

16. Canals M, Canals A. Percolación de la epidemia de influenza AH1N1 en el mundo: utilidad de los modelos predictivos basados en conectividad especial. Rev Med Chile 2010; 138: 573-80.

17. World Health Organization. 2010. http://www.who.int/ en/

18. Canals M. Predictibilidad a corto plazo de casos de influenza AH1N1 basado en modelos determinísticos. Rev Chil Infectol 2010; 27: 114-20.

19. Fraser C, Donnelly CA, Cauchemez S, Hanage WP, Van Kerkhove MD, Hollingsworth TD, et al. Pandemic Potential of a Strain of Influenza A (H1N1): Early Finding Science 2009; 324 (5934): 1557-61.

20. Patel M, Dennis A, Flutter C, Khan Z. Pandemic (H1N1) 2009 influenza. Br J Anaesthesia 2010; 104: 128-42.

21. Ministerio de Salud de Chile. 2010 http://www.minsal.cl (26-1-2010).

22. Heffernan JM, Smith RJ, Wahl LM. Perspectives on the basic reproductive ratio. J R Soc Interface 2005; doi: 10.1098/rsif.2005.0042: 1-13.

23. Mills CE, Robins JM, Lipsitch M. Transmisibility of 1918 pandemic influenza. Nature 2004; 432: 904-6.

24. Anderson RM. Epidemiology. En Modern Parasitology 
COX FEG ed Blackwell Scientific Publications, Oxford. 2003; 75-117.

25. Canals M, Cattan PE. Zoología médica: una visión de las especies potencialmente peligrosas desde la perspectiva de la biodiversidad. Generalidades y protozoos. Santiago, Chile: Editorial Universitaria; 2006, p. 160.

26. Bailey N. The mathematical theory of infectious diseases and its applications. London, England: Griffin; 1975. p. 413.

27. Canals M, Martínez L, Firinguetti L. Dinámica ecológica de enfermedades infecciosas. I parte: Estacionalidad. Rev Med Chile 1989; 117: 87-94.

28. Canals M. Patrones estacionales de enfermedades infecciosas: similitud y distancias. Rev Med Chile 1997; 125:
403-8.

29. Canals M, Bustamente RO, Ehrenfeld M, Cattan PE. Assesing the impact of disease vector son animal populations. Acta Biotheoretica 1998; 46: 337-45.

30. Canals M, Martínez L, Firinguetti L. Dinámica ecológica de enfermedades infecciosas. II parte: Recurrencia epidémica. Rev Med Chile 1989; 117: 95-100.

31. Centers for Disease Control and Prevention (CDC). Serum cross reactive antibody response to a novel influenza $\mathrm{A}(\mathrm{H} 1 \mathrm{~N} 1)$ virus after vaccination with seasonal influenza vaccine. MMWR Morb Mortal Wkly Rep 2009; 58: 521-4.

32. Cohen J. Past pandemics provide mixed clues to H1N1s next moves. www.sciencemag.org February 18, 2010. 